\title{
Is COVID-19 an opportunity to improve virtual leadership?
}

\author{
Deborah Claire Saltman
}

INTEGRATING EFFECTIVE virtual leadership activities into general practice should become a priority in the era of the COVID-19 pandemic. Despite the increase in remote working and the emergence of web-based clinical services, little is known about the effect of remote working on leadership and management, both in healthcare generally and in general practice specifically.

For the past six years I have been chairing a large committee of specialists, academics and public representatives and working virtually in an organisation that is responsible for one of the largest linked healthcare datasets in the world. ${ }^{1}$ Based on this experience, I believe that effective virtual leadership and management will vastly improve the remote landscape. In this article, I would like to share some of my observations about and practices for effective virtual leadership and management.

Even before the COVID-19 pandemic, remote working was on the rise. According to a Gartner poll, in the four years between 2012 and 2016 the number of people working remotely rose by $4 \%$. In the USA, $43 \%$ of workers spend some of their time working remotely. Remote work is a global phenomenon. In Finland, Japan, the Netherlands and Sweden, more than half the population work remotely for periods of time. ${ }^{2}$

The theoretical benefits to organisations of remote working are well documented in terms of efficiency, cost-effectiveness and flexibility. ${ }^{3}$ With the emergence of COVID-19, remote working has become essential to safeguard the health of all. However, the lack of face-to-face contact has a significant influence on productivity. ${ }^{4}$ Nearly half of virtual team respondents surveyed highlighted relationship building as the main contributor to the level of work productivity. ${ }^{3}$ Research in nursing suggests that virtual leadership lessens the impact of leadership styles but can improve personal connections among team members. ${ }^{5}$

A significant correlation was found between the overall emotional intelligence of the leader and the overall work engagement of virtual team members. ${ }^{6}$ This becomes more difficult when leaders and their teams are kept apart. The virtual environment requires a leader to establish new ways to stay connected with colleagues, and etiquettes that recognise the uniqueness of each individual. A virtual leader must carefully consider how to balance monitoring work output and giving employees sufficient autonomy.

Managing schedules within the infrastructure of the organisation is also important. For example, should part-time doctors and employees respond to texts or emails outside of their regular hours? Virtual management requires skills to handle tensions and dilemmas that cannot be resolved in person.

The complexity increases in general practice, where staff - for example nurses, receptionists and practice managers - are usually employees of an organisation, and the doctors are contractors. In practice, this results in the doctors expecting greater input into leadership and management.

The increased electronic traffic will require a management review of information technology (IT) infrastructure. Healthcare IT and virtual infrastructure are designed to protect confidentiality and transmit clinical and billing information, not management interaction between clinical and administrative staff. In general practice, IT is usually managed by contract agencies using off-the-shelf platforms with limited scope for changing rapidly. Therefore, reviewing and customising the applications to the new virtual management will require robust early, and continuing, review. Processes that will require review include assessment of whether the IT systems are: robust enough to handle the traffic of many more contemporaneous users, fit-for-purpose, user-friendly for meetings (and not too generic) and responsive in real time. Close relationships with IT contractors or departments and staff training also are critical.

In healthcare environments, ethical concerns are paramount. It is important to ensure that virtual management practices adhere to the codes of practice for data protection and confidentiality that are maintained in your organisation as well as your extended virtual environment. Leaders should ensure that staff working virtually understand how these issues apply, including the confidential storage of sensitive information when out of the office.

Very few managers or clinicians are trained in the virtual context. Specific attention needs to be paid to the in-themoment environment. As leaders we must adjust our styles to balance empathy with authority, diversity of opinion with guidance, coaching with prescribing. All this must achieved in an atmosphere in which signals are very different. Showing leadership at the outset is a good start. In online video or audio conferences, an effective leader will canvass all the participants to assure that every team member has an opportunity to contribute, 
not just those with the loudest voices. The leader will set the parameters of who speaks and for how long, as well as how much cross flow can occur between participants. Requiring speakers to raise their hands and be recognised will facilitate the conversation and ensure participants do not talk over one another. (It is best to 'go with the flow' but be prepared to bring the conversation back.)

Silences are perceived as being longer in virtual environments, and it is important to listen to what is said and what is not said. I use the seven-second rule (the average time a clinician allows for silence in a consultation), meaning that participants must wait at least seven seconds after someone has spoken before seeking to speak.

Dealing with conflicts can also be difficult. Evidence supports the concept of discussing relationships and conflicts with staff in a supportive way to enhance the virtual experience.

Agreement on the rules of engagement at the beginning is important. Highlighting points of agreement and progress throughout the conference and reframing issues can all help. ${ }^{7}$

The key to running an effective in-person meeting in a healthcare setting is keeping the focus from the first moment the objective is set, right up until the outcomes are ratified. It is the same for virtual meetings. Unfortunately, in the current environment the hallmarks of achieving this ideal cannot always be operationalised. For example, extensive pre-meeting planning and effective use of the meeting environment are not always possible. ${ }^{8}$ However, other components are transferrable. For example, objectives and outcomes must be in everyone's thoughts at all times during the virtual meeting. The temptation to do other things at the same time can be great. Attention to the flow of the meeting can help to reduce the anxiety that many of participants likely feel at this present time and provide a transparent, real-time forum for communication.

\section{Author}

Deborah Claire Saltman AM, PhD, MD, Chairman, Independent Advisory Steering Committee, Medicines \& Healthcare products, Regulatory Agency, UK; Visiting Professor, Imperial College, UK. debsaltman@gmail.com

Competing interests: None.

Funding: None.

Provenance and peer review: Commissioned, peer reviewed.

Citation: Saltman DC. Is COVID-19 an opportunity to improve virtual leadership? Aust J Gen Pract 2020;49 Suppl 12. doi: 10.31128/AJGP-COVID-12.

\section{References}

1. Clinical Practice Research Datalink: UK data driving real-world evidence. Clinical practice research datalink. London: CPRD, 2020. Available at www.cprd.com [Accessed 14 April 2020].

2. Rozwell C. How to cultivate effective 'remote work' programs. Stamford, CT: Gartner, 2018. Available at www.gartner.com/en/documents/3845967/ how-to-cultivate-effective-remote-work-programs [Accessed 14 April 2020].

3. Jarrell A. The experiences of team managers with knowledge sharing within diverse virtual teams [PhD thesis]. Minneapolis: Walden University, 2020.

4. Vogenberg FR, Santilli J. Healthcare trends for 2018. Am Health Drug Benefits 2018;11(1):48-54. [PhD thesis].

5. Duffy A. Nursing leadership and employee satisfaction perception in a virtual work [PhD thesis]. Minneapolis: Walden University, 2018.

6. Sebastian K, Hess J. Leader emotional intelligence and work engagement in virtual teams within a healthcare service setting: A quantitative study. Oklahoma State Medical Proceedings 2019;1(4).

7. Saltman DC, O'Dea NA, Kidd MR. Conflict management: A primer for doctors in training. Postgrad Med J 2006;82(963):9-12. doi: 10.1136/ pgmj.2005.034306.

8. O'Dea NA, de Chazal P, Saltman DC, Kidd MR. Running effective meetings: A primer for doctors. Postgrad Med J 2006;82(969):454-61. doi: 10.1136/pgmj.2005.042424. 